\title{
EDITORIAL AND COMMENT \\ Improving Shared Decision Making with LGBT Racial and Ethnic Minority Patients
}

\author{
Marshall H. Chin, MD, MPH', ${ }^{1,2}$ Fanny Y. Lopez, MPP ${ }^{7}$, Aviva G. Nathan, MPH', and Scott C. Cook, \\ $\mathrm{PhD}^{1,2}$ \\ 'Department of Medicine, Section of General Internal Medicine, University of Chicago, Chicago, IL, USA; ${ }^{2}$ Robert Wood Johnson Foundation \\ Finding Answers: Disparities Research for Change National Program Office, University of Chicago, Chicago, IL, USA.
}

KEY WORDS: shared decision making; communication; lesbian; gay; bisexual; transgender; race; ethnicity; disparities.

J Gen Intern Med 31(6):591-3

DOI: $10.1007 / \mathrm{s} 11606-016-3607-4$

(C) Society of General Internal Medicine 2016

$\mathrm{G}$ reat strides have been made in lesbian, gay, bisexual, and transgender (LGBT) rights and recognition. Gay marriage is supported by most of the U.S. population. Religious freedom laws that could lead to discrimination against LGBT persons in Indiana and Arkansas were rebuked by diverse organizations including Walmart, Apple, and Angie's List. Within healthcare, national efforts to report organizational competence in delivering care to LGBT patients, such as the Human Rights Campaign Healthcare Equality Index, have become important accreditations. ${ }^{1}$ Organizations such as the National LGBT Health Education Center have created curricula to help clinicians better care for LGBT patients. ${ }^{2}$

However, despite these advances, LGBT patients still suffer significant health disparities, often receive poor-quality care, and have negative health care experiences due to discrimination and lack of understanding of LGBT health issues and needs among health providers. ${ }^{3-5}$ LGBT patients frequently delay or avoid seeking care because they anticipate having negative experiences with health providers. ${ }^{3-5}$ Those who choose to receive medical care are often afraid of or feel uncomfortable disclosing their sexual orientation or gender identity to their health providers, which prevents them from having open and honest communication about their health care needs and preferences. Not disclosing this information to providers also often results in a lack of preventive services, inaccurate diagnosis, inappropriate treatment, and poor health outcomes in this population. ${ }^{3}$

LGBT patients who are also racial and ethnic minorities have an even higher risk of poor health outcomes, and many face more complicated challenges than white LGBT patients. ${ }^{4}$ For example, these patients are less likely to disclose their sexual orientation to health providers than those who are white. ${ }^{4}$ Some patients may have had experiences with persons in authority positions who did not understand how to deal with these statuses independently and together. For example, heightened cultural stigma about sexual and gender minorities

Published online March 17, 2016 in some racial/ethnic communities might make the consequences of disclosure worse than those for white LGBT patients, and prior bad experiences might affect the current provider-patient interaction. In addition, most resources in the LGBT community are geared toward white populations, and thus dual-minority patients may have a greater lack of appropriate information and support to empower them in their communication with providers.

Unfortunately, most clinicians do not have significant experience addressing issues at the intersection of LGBT and racial/ethnic minority statuses that impact communication and clinical care. They often lack the training and knowledge to encourage disclosure of sexual orientation and/or gender identity and to create a safe and welcoming environment. Clinicians often lack experience about how race and ethnicity further influence perceptions of and relationships with their LGBT patients. Even the most advanced mainstream healthcare organizations are often at only a basic level of LGBT cultural competence. We need to go beyond the basics of merely being inclusive and not offensive to patients, and begin addressing the specifics of how to improve communication between LGBT racial/ethnic minority patients and providers.

Data regarding the preferences of LGBT racial/ethnic minority patients around communication and shared decision making (SDM) are sparse. SDM, a fundamental component of patient-centered care, has been demonstrated to improve a variety of clinical outcomes, such as control of diabetes and hypertension. ${ }^{6}$ Therefore, in 2014, our team at the University of Chicago, supported by funds from the Agency for Healthcare Research and Quality and the Patient-Centered Outcomes Research Trust Fund, began examining how to reduce disparities for LGBT racial/ethnic minority patients through improved SDM. We have three goals for improving SDM with LGBT racial/ethnic minority groups: 1) review what is known, 2) perform interviews and focus groups of patients and clinicians, and 3) develop tools and resources.

The three articles in this month's JGIM symposium on "Improving Shared Decision Making with LGBT Racial and Ethnic Minority Patients" reflect our initial foundational work based on literature review from the fields of medical decision making, race/ethnicity, gender studies, and implementation science. $^{6-8}$ The three papers outline a conceptual model for 
SDM with LGBT racial/ethnic minority patients, and then present information that may guide interventions to assist individual clinicians and patients in improving their SDM, and may help health care organizations transform their structures to facilitate SDM.

SDM is defined as communication and interaction between clinicians and patients that incorporate information sharing, discussion of care options and elicitation of patient values and preferences, and decision making. LGBT status includes multiple dimensions, of which two of the most important are sexual orientation and gender identity. Sexual orientation has three components: 1) the emotional and physical attraction to others of a particular sex, on a continuum from exclusively heterosexual to exclusively homosexual; 2) self-reported identity (which may differ from innate attraction or behavior); and 3) sexual behavior (which may differ from attraction and/or identity). Gender identity is a complex concept that is often distilled to a person's innate and inescapable perception of their own gender, which may or may not be consistent with the person's anatomical sex or sex assigned at birth. For many people, these components of sexual and gender identity are dynamic and fluid.

Peek et al. present a conceptual model for SDM in LGBT racial/ethnic minority patients, with a focus on LGBT AfricanAmerican patients. ${ }^{6}$ They elucidate the intersection of LGBT status and race by integrating and expanding Peek's adaptation of the Charles model of SDM for African Americans with the Model of Multiple Dimensions of Identity. ${ }^{6}$ Key insights include the influence of each individual's perception of himself or herself, given his or her intersecting identity axes of sexual orientation, gender identity, race, and ethnicity; the clinician's perception of the patient, given these identities and vice versa; and the influence of social networks and society. Understanding these complex inter- and intra-personal dynamics is critical for engaging in effective, empathetic communication and SDM.

To illustrate the implications of this intersectionality framework, our team presented a workshop on improving SDM with LGBT racial/ethnic minority patients at the 2015 Society of General Internal Medicine Annual Meeting, in which participants engaged in four case role plays. ${ }^{9}$ One SDM vignette about pre-exposure HIV prophylaxis medication features an African-American man who selfidentifies as heterosexual, has sex with women and men, and is a member of a predominantly African-American social network distinct from the gay white male networks that typically receive HIV prevention and education resources. Another case presents the challenge of discussing Pap smear screening with a Hispanic transgender man who is ambivalent about having a test associated with women. A third vignette presents an African-American woman with cognitive impairment and her lesbian partner who come to the clinic to discuss advanced care planning, in the context that the patient's son believes homosexuality is a sin. The last vignette features an undocumented
Latina woman who is the victim of intimate partner violence from her lesbian partner, who is threatening to "out" her if she leaves. Each case raises difficult issues for clinicians to navigate with their patients at the intersection of LGBT status and race/ethnicity.

One way to help this navigation might be decision aids, tools to improve the quality of SDM between clinicians and patients. In a systematic review, Nathan et al. found that decision aids have improved communication and psychological outcomes in racial/ethnic minority populations. ${ }^{7}$ However, they identified only one study of decision aids in sexual minorities and none for transgender patients.

While research and education about SDM have tended to focus on the individual clinician-patient encounter, the structure of the care setting can greatly impact the likelihood that SDM occurs and whether the SDM is of high quality. DeMeester et al. examine what organizations can do to improve SDM with LGBT racial/ethnic minority patients, and identify fundamental drivers and mechanisms for organizational systems solutions. ${ }^{8}$ Drivers of SDM encompass workflows, health information technology, organizational structure and culture, resources and clinic environment, training and education, and rewards and disincentives. Mechanisms by which these drivers impact SDM include the continuity and coordination of care, ease of SDM, knowledge and skills of clinicians and staff, and attitudes and beliefs of the organization's personnel.

Despite societal advances in the ways LGBT persons are viewed and treated, health care disparities among LGBT racial/ ethnic minority persons remain an important, largely hidden problem, causing unwarranted ill health effects and suffering. The crucial steps toward improving SDM with these highly marginalized patients are as follows: increasing clinicians' awareness and skills in addressing issues at the intersection of sexual orientation, gender identity, and racial/ethnic minority status; empowering patients to be active participants in their care; and making the clinical environment culturally competent and conducive to SDM. We hope our conceptual framework, review of patient decision aids, and model of organizational context provide a foundation to accomplish these goals. With ongoing qualitative research, we will interview clinicians and LGBT racial/ethnic minority patients about their experiences, needs, and recommendations regarding SDM across a variety of clinical topics that include anal cancer screening, HIV preexposure prophylaxis (PrEP), hepatitis $\mathrm{C}$, diabetes, obesity and body image, mental health, gender transition and primary care for transgender persons, intimate partner violence, and older adult decision making. Future findings can be incorporated into road maps designed to help health organizations reduce health care disparities. ${ }^{10}$ Addressing issues at the intersection of LGBT and racial/ethnic minority statuses expands and complicates what it means to be culturally competent and inclusive. As such, research, education, and the implementation of findings to 
improve SDM in LGBT racial/ethnic minority patients must be priorities.

Acknowledgments: This project was supported by the Agency for Healthcare Research and Quality (1U18 HSO23050) and the Robert Wood Johnson Foundation Finding Answers: Disparities Research for Change Program. Dr. Chin was also supported by a National Institute of Diabetes and Digestive and Kidney Diseases Midcareer Investigator Award in Patient-Oriented Research (K24 DK071933) and the Chicago Center for Diabetes Translation Research (P30 DK092949). Some of the paper's content was presented as a workshop at the Society of General Internal Medicine Annual Meeting in Toronto, Canada, April 23, 2015.

Corresponding Author: Marshall H. Chin, MD, MPH; Department of Medicine, Section of General Internal Medicine, University of Chicago, 5841 South Maryland Avenue, MC 2007, Chicago, IL 60637, USA (e-mail: mchin@medicine.bsd.uchicago.edu).

Compliance with Ethical Standards:

Conflict of Interest: The authors declare that they do not have a conflict of interest.

\section{REFERENCES}

1. Human Rights Campaign Healthcare Equality Index. Available at: http:// www.hrc.org/campaigns/healthcare-equality-index. Accessed January 12 , 2016.

2. National LGBT Health Education Center. Available at: http://www. lgbthealtheducation.org/. Accessed January 12, 2016.
3. Mail PD. The case for expanding educational and community-based programs that serve lesbian, gay, bisexual, and transgender populations. Clin Res Regul Aff. 2002;19(2-3):223-73.

4. Wilson PA, Yoshikawa H. Improving access to health care among AfricanAmerican, Asian and Pacific Islander, and Latino lesbian, gay, and bisexual populations. In: Meyer IH, Northridge ME, eds. The Health of Sexual Minorities: Public Health Perspectives on Lesbian, Gay, Bisexual, and Transgender Populations. New York: Springer US; 2007:607-37.

5. Gay and Lesbian Medical Association. Guidelines for Care of Lesbian, Gay, Bisexual, and Transgender Patients. Gay and Lesbian Medical Association (GLMA), 2006. Available at: http://glma.org/_data/n_0001/resources/ live/GLMA\%20guidelines\%202006\%20FINAL.pdf. Accessed January 12, 2016.

6. Peek ME, Lopez FY, Williams HS, et al. Development of a conceptual framework for understanding shared decision-making among AfricanAmerican LGBT patients and their clinicians. J Gen Intern Med. doi:10. 1007/s11606-016-3616-3.

7. Nathan AG, Marshall IM, Cooper JM, Huang ES. Use of decision aids with minority patients: a systematic review. J Gen Intern Med. doi: 10.1007/s11606-016-3609-2.

8. DeMeester R, Lopez FY, Moore JE, Cook SC, Chin MH. A model of organizational context and shared decision making: application to LGBT racial and ethnic minority patients. J Gen Intern Med. doi:10.1007/ s11606-016-3608-3.

9. Case vignettes for workshop "Meeting the Health Care Needs of Racially Diverse LGBT Patients." Society of General Internal Medicine 2015 Annual Meeting. Toronto, Ontario. Available at: http://www.sgim.org/ File\%20Library / SGIM / Meetings/Annual\%20 Meeting/ Meetign\%20Content/AM15\%20handouts/WD03-Chin-Handout.pdf. Accessed January 12, 2016.

10. Chin MH, Clarke AR, Nocon RS, et al. A roadmap and best practices for organizations to reduce racial and ethnic disparities in health care. J Gen Intern Med. 2012;27:992-1000. 